\title{
Systematic review and network meta-analysis of resective surgery for mesial temporal lobe epilepsy
}

\author{
Puneet Jain, ${ }^{1}$ George Tomlinson, ${ }^{2,3}$ Carter Snead, ${ }^{1}$ Beate Sander, ${ }^{2,3}$ Elysa Widjaja ${ }^{1,3,4}$
}

- Additional material is

published online only. To view please visit the journal online (http://dx.doi.org/10.1136/ jnnp-2017-317783).

${ }^{1}$ Epilepsy Program, Division of Neurology, Department of Pediatrics, The Hospital for Sick Children, Toronto, Ontario,

Canada

${ }^{2}$ Toronto Health Economics and Technology Assessment (THETA), University Health Network,

Toronto, Ontario, Canada ${ }_{3}^{3}$ Institute of Health Policy, Management and Evaluation, University of Toronto, Toronto, Ontario, Canada

${ }^{4}$ Diagnostic Imaging, The Hospital for Sick Children, Toronto, Ontario, Canada

Correspondence to Dr Elysa Widjaja, Diagnostic Imaging, Hospital for Sick Children, Toronto, Ontario ON M5G 1X8, Canada; Elysa. Widjaja@sickkids.ca

Received 7 December 2017 Revised 5 March 2018 Accepted 22 April 2018 Published Online First 16 May 2018

Check for updates

To cite: Jain $\mathrm{P}$, Tomlinson $\mathrm{G}$, Snead C, et al. J Neurol Neurosurg Psychiatry 2018:89:1138-1144.

\section{ABSTRACT \\ Objective To evaluate the effectiveness of} anterior temporal lobectomy (ATL) versus selective amygdalohippocampectomy (SAH) on seizure-free outcome in patients with temporal lobe epilepsy, using both direct and indirect evidence from the literature. Methods MEDLINE, Embase and Cochrane databases were searched for original research articles and systematic reviews comparing ATL versus $S A H$, and ATL or SAH versus medical management (MM). The outcome was seizure freedom at 12 months of follow-up or longer. Direct pairwise meta-analyses were conducted, followed by a random-effect Bayesian network metaanalysis (NMA) combining direct and indirect evidence. Results Twenty-eight articles were included (18 compared ATL vs SAH, 1 compared ATL vs SAH vs MM, 8 compared ATL vs MM, and 1 compared SAH vs MM). Direct pairwise meta-analyses showed no significant differences in seizure-free outcome of ATL versus SAH (OR 1.14, 95\% Cl 0.93 to 1.39; $p=0.201$ ), but the odds of seizure-free outcome were higher for ATL versus MM (OR 29.16, 95\% Cl 10.44 to 81.50; $p<0.00001$ ), and SAH versus MM (OR $28.42,95 \% \mathrm{Cl} 10.17$ to 79.39 ; $p<0.00001)$. NMA also showed that the odds of seizurefree outcome were no different in ATL versus SAH (OR $1.15,95 \%$ credible interval (Crl) $0.84-1.15)$, but higher for ATL versus MM (OR 27.22, 95\% Crl 15.38-27.22), and SAH versus MM (OR 23.57, 95\% Crl 12.67-23.57). There were no significant differences between direct and indirect comparisons (all p>0.05).

Conclusion Direct evidence, indirect evidence and NMA did not identify a difference in seizure-free outcome of ATL versus SAH.

\section{INTRODUCTION}

Temporal lobe epilepsy (TLE) is the most common cause of drug refractory epilepsy, especially in adults. Eighty per cent of TLEs have onset in the hippocampus, ${ }^{1}$ and hippocampal sclerosis is a major aetiology in the adult epilepsy surgery series. Advances in neuroimaging and neurophysiology have enabled more precise localisation of the epileptogenic zone and the eloquent areas, thus helping in planning surgical resection. Two randomised controlled trials have established the superiority of epilepsy surgery over medical management (MM) for patients with TLE. $^{2}{ }^{3}$ Standardised anterior temporal lobectomy (ATL) involves removing $4-6 \mathrm{~cm}$ of the anterior temporal lobe, including the amygdala and hippocampus. Selective amygdalohippocampectomy (SAH), the other common surgery for TLE, preserves the temporal neocortex and underlying white matter, and offers theoretical advantage of lower cognitive decline following surgery. The choice of resective surgery for TLE remains elusive. Previous meta-analyses have shown discordant findings, with ATL reported to achieve better seizure outcome when compared with SAH in two meta-analyses, ${ }^{45}$ and similar seizure outcome was reported with ATL and SAH in another meta-analysis. ${ }^{6} \mathrm{Hu}$ et $a l^{4}$ also showed that there was no significant difference in fullscale performance and verbal IQ scores of ATL relative to SAH. Further, all three meta-analyses were conducted using standard pairwise comparison of ATL versus SAH.

Network meta-analysis (NMA) has the advantage of incorporating both direct and indirect evidence. Direct evidence compared ATL relative to SAH; indirect evidence compared the relative effect of either of these two temporal lobe surgeries with the common comparator, that is, MM. NMA includes multiple pairwise comparisons across a range of interventions, that is, ATL versus SAH, ATL versus $\mathrm{MM}$, and $\mathrm{SAH}$ versus $\mathrm{MM}$, and provides estimates of the relative effect on multiple treatment comparisons. ${ }^{7}$ The aim of this systematic review and NMA was to evaluate the effectiveness of ATL versus SAH on seizure-free outcome, using both direct and indirect evidence from the literature.

\section{METHODS}

\section{Search strategy}

This systematic review was conducted in accordance with the Preferred Reporting Items for Systematic Reviews and Meta-Analyses guideline. MEDLINE, Embase and the Cochrane databases were searched systematically (on 27 June 2017) using the key terms 'temporal lobe epilepsy', 'anterior temporal lobectomy' and 'amygdalohippocampectomy'. The search strategy is detailed in online supplementary table 1 . We also screened the reference section of the included articles for any additional relevant studies.

Original research articles (experimental or observational) and systematic reviews comparing ATL versus SAH, ATL versus MM, or SAH versus MM for TLE, with at least 12 months of follow-up, were included. Searches were restricted to English-language articles published from 1990 to 2017. If there were multiple studies reporting on overlapping patient populations from the same centre, the most recent article was used. Exclusion criteria were non-human 
studies, grey literature, conference abstracts, case reports and editorials.

\section{Data extraction}

Two authors (PJ and EW) independently extracted the data including author, year of publication, country of origin, interventions used, study design, criteria for choosing a particular intervention, investigations for presurgical evaluation, age at the time of surgery or study enrolment, duration of epilepsy, side of surgery, duration of follow-up, sample size, classification used for seizure outcome and seizure-free outcome. Any disagreement in the extracted data was resolved by reaching a consensus through discussion. Where the relevant data were missing, we contacted the corresponding author for further details.

\section{Outcome measure}

The outcome was seizure freedom on follow-up at 12 months or longer. If the seizure outcome was reported at multiple prespecified time points, outcome at 2 years was used for the meta-analysis and NMA.

\section{Data analysis}

Meta-analyses: direct pairwise comparisons

RevMan (Review Manager) V.5.2 was used for direct pairwise meta-analyses. ${ }^{8}$ Three separate meta-analyses were done, comparing ATL versus SAH, ATL versus MM, and SAH versus $\mathrm{MM}$. The data from various studies were pooled and expressed as OR with $95 \%$ CI. A p value $<0.05$ was considered statistically significant. We used the $\mathrm{Q}$ statistic and $\mathrm{I}^{2}$ to test for betweenstudy heterogeneity. If statistically significant heterogeneity was present ( $Q$ statistic $\mathrm{p}<0.05$ or $\mathrm{I}^{2} \geq 50 \%$ ), the pooled estimate and 95\% CI were calculated using a random-effect model. If there was no significant heterogeneity between studies, the fixed-effect model was used. Publication bias was assessed using funnel plot. The funnel plot was visually assessed for asymmetry, which is indicative of potential publication bias. Assessment of publication bias was conducted if there were at least three studies for each pairwise comparison.

Subanalyses were conducted for ATL versus SAH for studies reporting on surgery for hippocampal sclerosis only; studies reporting on seizure-free outcome at 1-year, 2-year and 5-year follow-up; studies reporting complete seizure freedom and free from disabling seizures; and studies published before and after 2000 .

\section{Network meta-analysis}

A random-effect NMA combining direct and indirect evidence was performed within a Bayesian framework ${ }^{9}$ using R V.3.3.3, gemtc package. Consistency of direct and indirect evidence was assessed using node-splitting models. The consistency results were considered insignificant when the $\mathrm{p}$ value was greater than 0.05 for the comparison between direct and indirect effects in the node-splitting analysis. Model convergence was assessed using the Gelman-Rubin statistics, and convergence was considered to have been achieved if the widths of pooled runs and individual runs stabilised around the same value and their ratio was approximately 1 .

\section{Quality of evidence}

The GRADE Profiler software (V.3.2) was used to assess the quality of evidence included in the meta-analyses. The following were considered in the Grading of Recommendations, Assessment, Development and Evaluations (GRADE) criteria ${ }^{10}$ : study design, risk of bias, inconsistency, indirectness, imprecision, publication bias, magnitude of effect, dose response gradient and residual confounding. The quality of evidence was classified as very low, low, moderate or high.

The quality of previous systematic reviews was assessed by the Assess the Methodological Quality of Systematic Review (AMSTAR) tool. ${ }^{11}$

\section{RESULTS}

Of the 4696 citations identified, full texts of 51 articles were reviewed for eligibility (figure 1). Twenty-eight studies were included in this systematic review. Twenty-three studies were excluded as the outcomes of interests were not reported, ${ }^{12-27}$ there was overlapping patient population, ${ }^{28-30}$ unclear nature of surgery, ${ }^{31} 32$ or the follow-up was less than 1 year. ${ }^{33} 34$

\section{Study characteristics}

The study characteristics are summarised in online supplementary table 2. The studies were from the USA (8), Canada (5), Brazil (4), Germany (4), UK (4), France (1), Japan (1), Australia (1) and Pakistan (1). All the included studies were observational studies except for two randomised controlled trials. ${ }^{2}$ Four were prospective studies ${ }^{35-38}$ and the rest were retrospective or had unknown study designs. Of the 28 articles included, 18 studies provided direct pairwise comparison of ATL versus SAH, ${ }^{35} 36$ 39-54 1 study compared three interventions (ATL, SAH and MM), ${ }^{55} 8$ studies compared ATL versus MM, $33856-60$ and 1 study compared SAH versus MM. ${ }^{37}$ In 17 studies, the outcome was seizure freedom (or specified using Engel Ia or International League against Epilepsy (ILAE) class I), and in 12 studies the outcome was freedom from disabling seizures.

The SAH was done through transcortical (nine studies), trans-sylvian (seven studies) and subtemporal (two studies) approaches. In most studies, selection of the surgical procedure was based on time period, with SAH being conducted later in the study period compared with ATL, surgeon's expertise or preference. Majority of the studies enrolled adult patients except one. ${ }^{44}$ The mean duration of epilepsy was predominantly greater than 10-20 years, with the exception of the Early Randomized Surgical Epilepsy Trial, ${ }^{2}$ where the median duration of epilepsy was 5.2 years. Hippocampal sclerosis was the exclusive aetiology in 12 articles. $^{3637404145474852-545760}$

\section{Previous systematic reviews}

We found three systematic reviews and meta-analyses ${ }^{4-6}$ comparing ATL versus SAH. The odds of seizure-free outcome were lower for SAH relative to ATL in one systematic review, ${ }^{4}$ and the relative risk of seizure-free outcome was higher for ATL relative to SAH in another systematic review ${ }^{5}$ (see online supplementary table 3). One systematic review did not identify significant differences in the relative risk of seizure-free outcome of ATL versus SAH. ${ }^{6}$ The detailed AMSTAR scoring is tabulated in online supplementary table 4.

\section{Meta-analyses: direct pairwise comparisons ATL versus SAH}

There were 19 studies comparing ATL versus SAH. There was no significant difference in seizure-free outcome following ATL relative to SAH (OR $1.14,95 \%$ CI 0.93 to $1.39 ; \mathrm{p}=0.20$ ) (figure 2). The funnel plot (figure 3A) showed no evidence of publication bias. The quality of evidence was 'very low' (online supplementary table 5). 


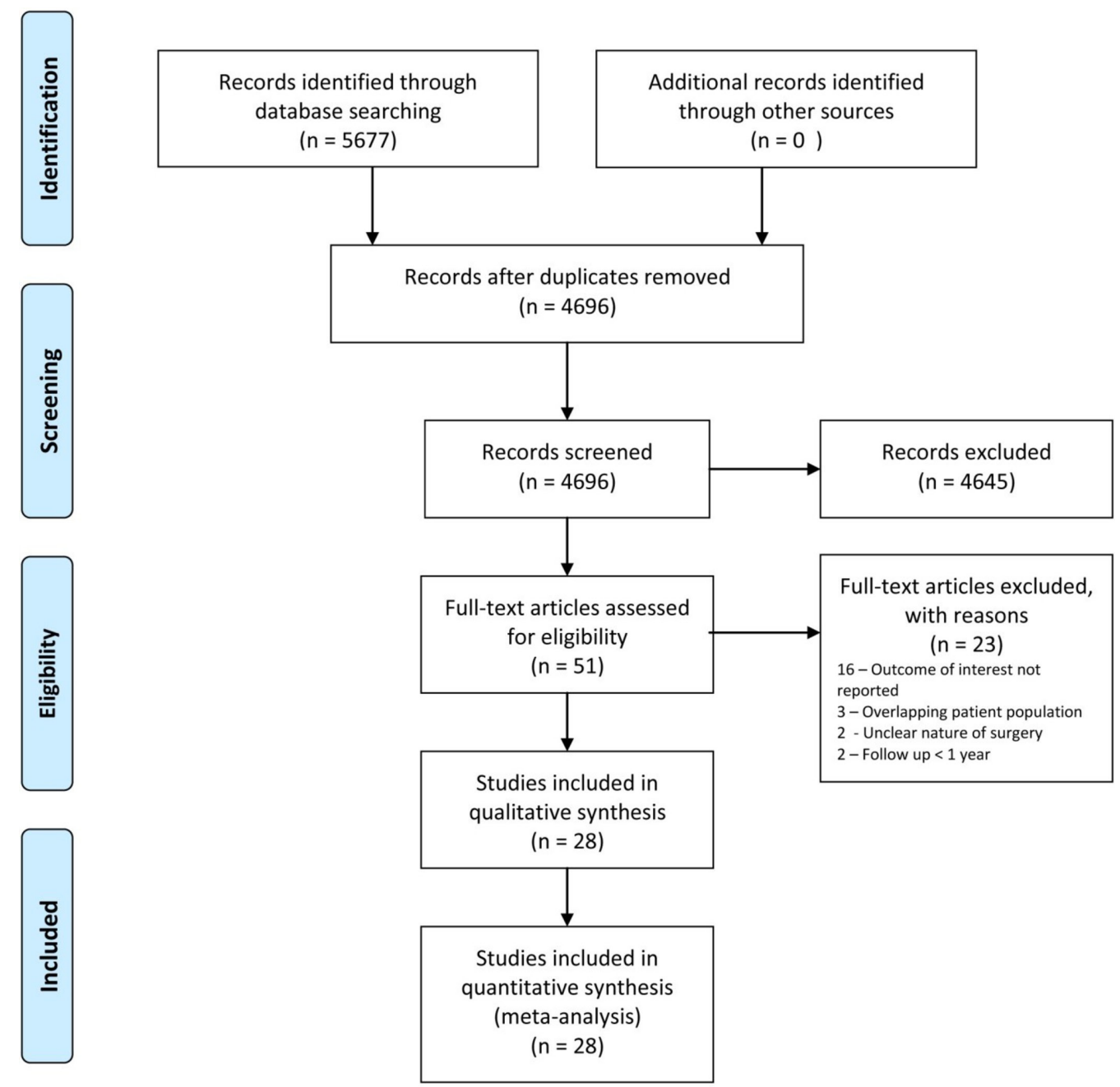

Figure 1 PRISMA flow chart of study selection and reasons for exclusion. PRISMA, Preferred Reporting Items for Systematic Reviews and Meta-Analyses.

Subgroup analysis (online supplementary table 6) of studies that included patients with hippocampal sclerosis only ${ }^{36} 404145474852-54$ showed no significant difference in the seizure-free outcome of ATL versus SAH (OR 1.05, 95\% CI 0.80 to $1.37 ; \mathrm{p}=0.75)$. Subgroup analyses of studies that reported on seizure-free outcome at 1 -year (OR $1.08,95 \%$ CI 0.82 to 1.41 ; $\mathrm{p}=0.58$ ), 2-year (OR 1.48, 95\% CI 0.97 to $2.27 ; \mathrm{p}=0.07$ ) and 5 -year (OR $1.49,95 \%$ CI 0.82 to $2.70 ; \mathrm{p}=0.19$ ) follow-up also did not show significant differences in seizure-free outcome of ATL versus SAH. Studies reporting complete seizure freedom (OR $0.95,95 \%$ CI 0.73 to $1.24 ; \mathrm{p}=0.72$ ) and studies published before 2000 (OR 1.10, 95\% CI 0.83 to 1.46 ; p = 0.52) and after 2000 (OR 1.36, 95\% CI 0.89 to $2.09 ; \mathrm{p}=0.15$ ) did not show any significant difference in seizure outcome of ATL versus SAH (online supplementary table 6). However, studies reporting freedom from disabling seizure showed that ATL had higher odds of freedom from disabling seizure compared with $\mathrm{SAH}$ (OR $1.48,95 \%$ CI 1.16 to $1.87 ; \mathrm{p}=0.001$ ).

\section{ATL versus MM}

There were nine studies comparing ATL versus MM. The odds of seizure-free outcome were significantly higher for ATL as compared with MM (OR 29.16, 95\% CI 10.44 to 81.50 ; $\mathrm{p}<0.00001$ ) (figure 4). The funnel plot (figure 3B) showed evidence for publication bias, with fewer studies on the right of the funnel plot, suggesting that the effect size would have been smaller if there were more studies on the right of the funnel plot. The quality of evidence for the nine studies was 'very low'.

\section{SAH versus MM}

Two studies compared SAH versus MM. The odds of seizurefree outcome were also significantly higher for SAH as compared with MM (OR 28.42, 95\% CI 10.17 to 79.39 ; p <0.00001) (figure 5). The quality of evidence for these two studies was 'very low'.

\section{Network meta-analysis}

The network plot (online supplementary figure 1) shows that the strength of comparison, as reflected by the edges linking the treatment strategies, was greatest for ATL versus SAH, followed by ATL versus MM, and weakest for SAH versus MM. NMA showed that there was no difference in the odds of seizure-free outcome of ATL versus SAH (OR 1.15, 95\% credible interval (CrI) 0.84-1.15). The odds of seizure-free outcome were higher for ATL versus MM (OR 27.22,95\% CrI 15.38-27.22) and also for SAH versus MM (OR 23.57, 95\% CrI 12.67-23.57). There were no significant differences between direct and indirect comparisons for the three pairwise comparisons (ATL vs $\mathrm{SAH}$, ATL vs MM, and SAH vs MM) (all p>0.05) (figure 6). The Gelman diagnostic indicated that the model converged (all 1.00). 


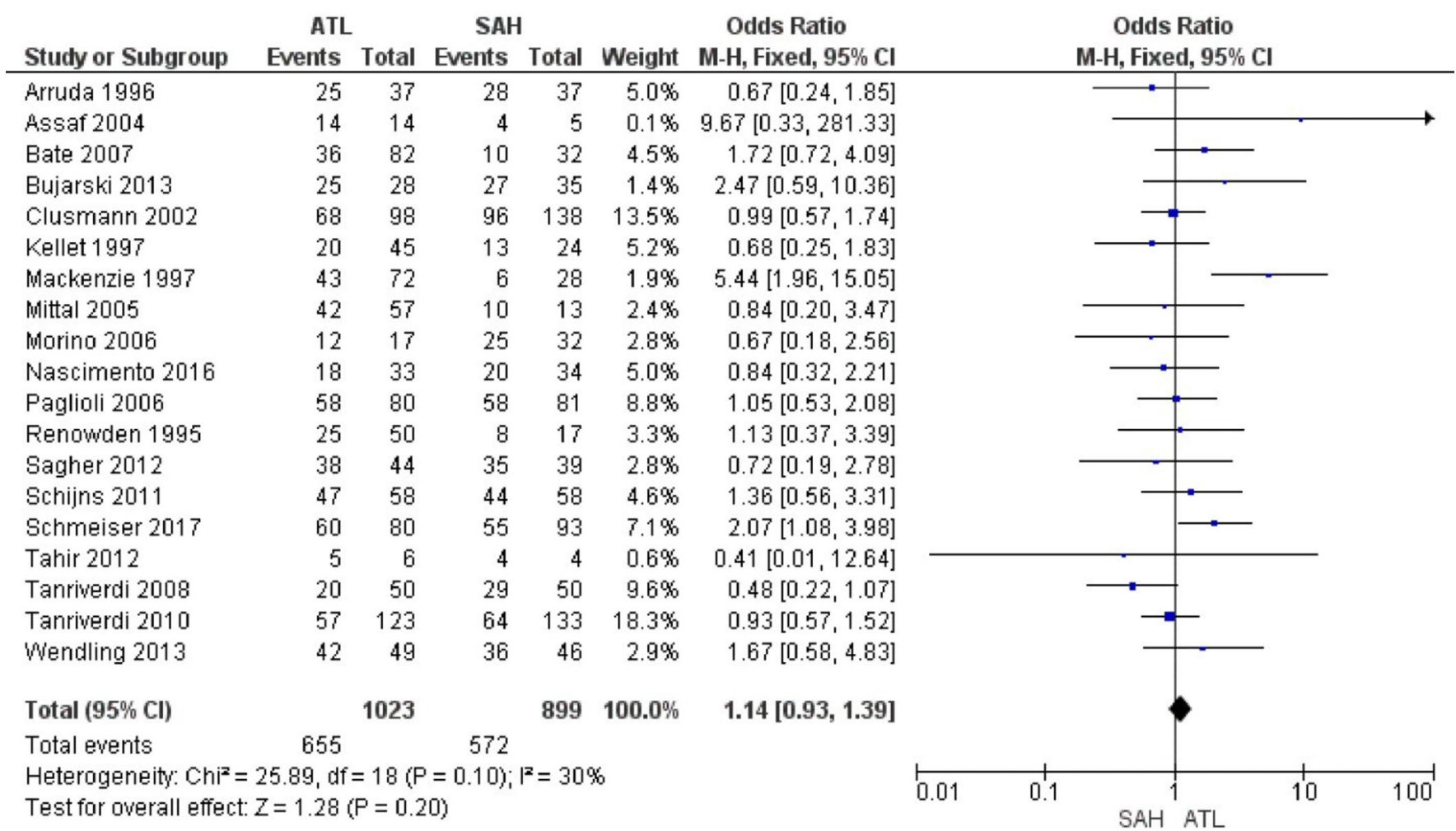

Figure 2 Forest plot comparing anterior temporal lobectomy (ATL) and selective amygdalohippocampectomy (SAH) for seizure-free outcome. M-H, MantelHaenszel.

\section{DISCUSSION}

Existing meta-analyses have used direct pairwise comparison of ATL versus SAH. The study by Josephson et $a l^{5}$ included 11 articles in their meta-analysis and found that the relative risk of freedom from disabling seizures was 1.3 times higher with ATL compared with SAH. In the meta-analysis conducted by $\mathrm{Hu}$ et $a l,{ }^{4}$ the authors also included 11 articles in their meta-analysis and demonstrated that the odds of seizure freedom were 0.65 times lower for SAH compared with ATL. Kuang et $a l^{6}$ did not find a significant difference in seizure control rate 1 year after surgery, but only included six articles in their meta-analysis. The difference in findings of these meta-analyses could be related to the inclusion of different number of studies, studies with different follow-up duration or restricting the studies to those with 1-year follow-up. There were more studies that were included in our meta-analysis compared with prior meta-analyses by Josephson $e t a l^{5}$ and $\mathrm{Hu}$ et $a l .{ }^{4}$ In particular, there were seven studies that were not included in prior meta-analyses-Arruda et $a l,{ }^{39}$ Renowden et al, ${ }^{48}$ Tahir et al, ${ }^{51}$ Sagher et al, ${ }^{49}$ Bujarski et $a l^{41}$ Wendling et $a l^{54}$ and Nascimento et $a l^{46}$ - which demonstrated no significant difference in seizure-free outcome of ATL versus SAH. The inclusion of new data from these studies may have contributed to differences in this study's conclusion relative to prior meta-analyses.

This study combined both direct and indirect evidence, which may strengthen the assessment of direct pairwise comparison of ATL versus $\mathrm{SAH}^{61}$ as both direct and indirect evidence contribute to the total body of evidence. Combining direct and indirect evidence may avoid bias from omission of data, and can give more precise estimates of treatment effects. ${ }^{9}$ In our study, we have included 19 articles with direct pairwise comparison of ATL versus SAH, and 10 articles (one of the articles compared
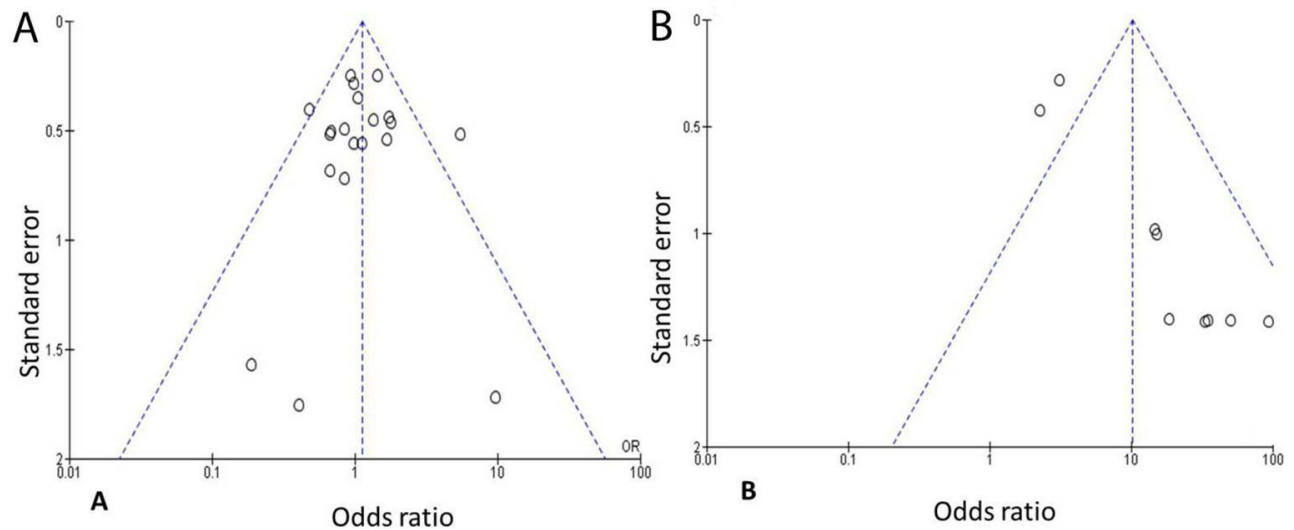

Figure 3 Funnel plots of anterior temporal lobectomy (ATL) versus selective amygdalohippocampectomy (A), and ATL versus medical management (B). 


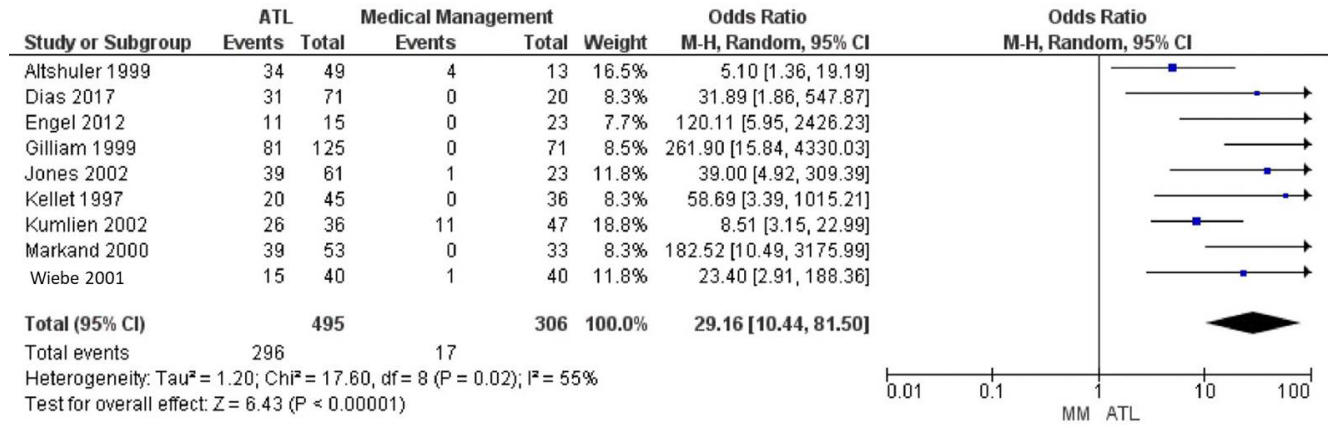

Figure 4 Forest plot showing the comparison between anterior temporal lobectomy (ATL) and medical management (MM) for seizure-free outcome. M-H, Mantel-Haenszel.

ATL vs SAH vs MM) provided indirect comparison of ATL or SAH with MM. By including more evidence in the pairwise analysis compared with the study by Josephson et $a l^{5}$ and $\mathrm{Hu}$ et $a l^{4}{ }^{4}$ we showed that ATL did not achieve significantly better seizure-free outcome relative to SAH. Further, we demonstrated that direct and indirect evidence, as well as NMA, all reached the same conclusion, in that ATL was not superior to SAH in achieving seizure-free outcome. It is possible that improvement in presurgical evaluation, including high-resolution structural and functional imaging, and technical advances in electroencephalography (EEG), may have contributed to improved selection of candidates for SAH.

The criteria for selecting the surgical technique (ATL vs $\mathrm{SAH}$ ) in the articles were variable and were frequently based on institutional preference and expertise of the neurosurgeon. Few studies also reported preferred use of SAH if the interictal/ ictal EEG findings were restricted to the mesial temporal lobe. A previous randomised controlled trial comparing $2.5 \mathrm{~cm}$ vs $3.5 \mathrm{~cm}$ mesial temporal resection in TLE found no difference in seizure freedom rate for the $2.5 \mathrm{~cm}$ resection relative to $3.5 \mathrm{~cm}$ resection group, suggesting that adequate volume resection rather than maximal volume resection is more important for good seizure outcome. ${ }^{62}$ Given that complete resection of the epileptogenic zone plays an important role in determining the success of epilepsy surgery, there could be a preference for SAH if the interictal/ictal EEG findings were localised to the mesial temporal lobe. There are various surgical approaches for SAH, such as trans-sylvian, transcortical/transtemporal and subtemporal approach, which have been postulated to have different theoretical advantages, ${ }^{63}$ and the use depends on surgeons' experience and skills. Studies comparing these approaches are limited and showed similar seizure outcome. ${ }^{486465}$

There is lack of consensus in the literature regarding the neuropsychological outcomes following ATL and SAH. ${ }^{63}$ The standardised mean differences in verbal IQ, performance IQ and full-scale IQ following ATL and SAH were found to be not significant in a meta-analysis. ${ }^{4}$ Schramm ${ }^{66}$ showed that 11 out of 14 articles on SAH reported better cognitive outcome. Tanriverdi et $a l^{53}$ reviewed cognitive outcome after temporal lobe surgery and found that 16/21 studies demonstrated better cognitive outcome after SAH, compared with 5/21 studies which showed no difference. Due to the lack of standardised testing and reporting of neuropsychological outcome in the literature, we did not undertake a meta-analysis of neuropsychological outcome following ATL versus SAH. Neuropsychological outcomes following temporal lobe surgery are dependent on factors other than the type of surgery, including cortical eloquent sites for language or memory, ${ }^{67}$ pathological status (presence or absence of hippocampal sclerosis) ${ }^{68}$ and age at seizure onset, with older age at seizure onset associated with worse outcome. ${ }^{69}$

There are several limitations to this systematic review and meta-analyses. All direct evidence, that is, pairwise comparison of ATL versus SAH, were observational studies and were of 'very low' quality evidence. Similarly, all except two indirect evidence, that is, pairwise comparison of ATL or SAH versus MM, were observational studies and were of 'very low' quality evidence. Another limitation of this study is the variability in the definition of seizure-free outcome. Over half of the articles used complete seizure freedom to infer seizure-free outcome, while just under half of the articles used freedom from disabling seizures as the outcome measure. Heterogeneity with respect to duration of follow-up could potentially confound the findings. We have conducted subgroup analyses and showed that seizurefree outcomes at 1-year, 2-year and 5-year follow-up were not significantly different between ATL and SAH. There was also heterogeneity in aetiologies, criteria for selection of surgical procedures and surgical approach for SAH. A randomised controlled trial comparing ATL versus SAH will overcome many of the methodological limitations of observational study, and could include seizure freedom as an outcome measure, and other

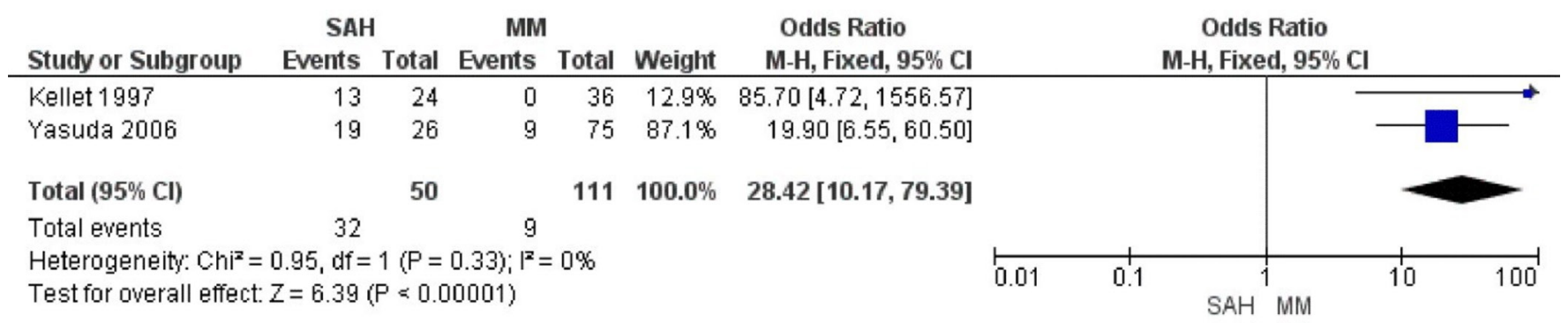

Figure 5 Forest plot showing the comparison between selective amygdalohippocampectomy (SAH) and medical management (MM) for seizure-free outcome. M-H, Mantel-Haenszel. 


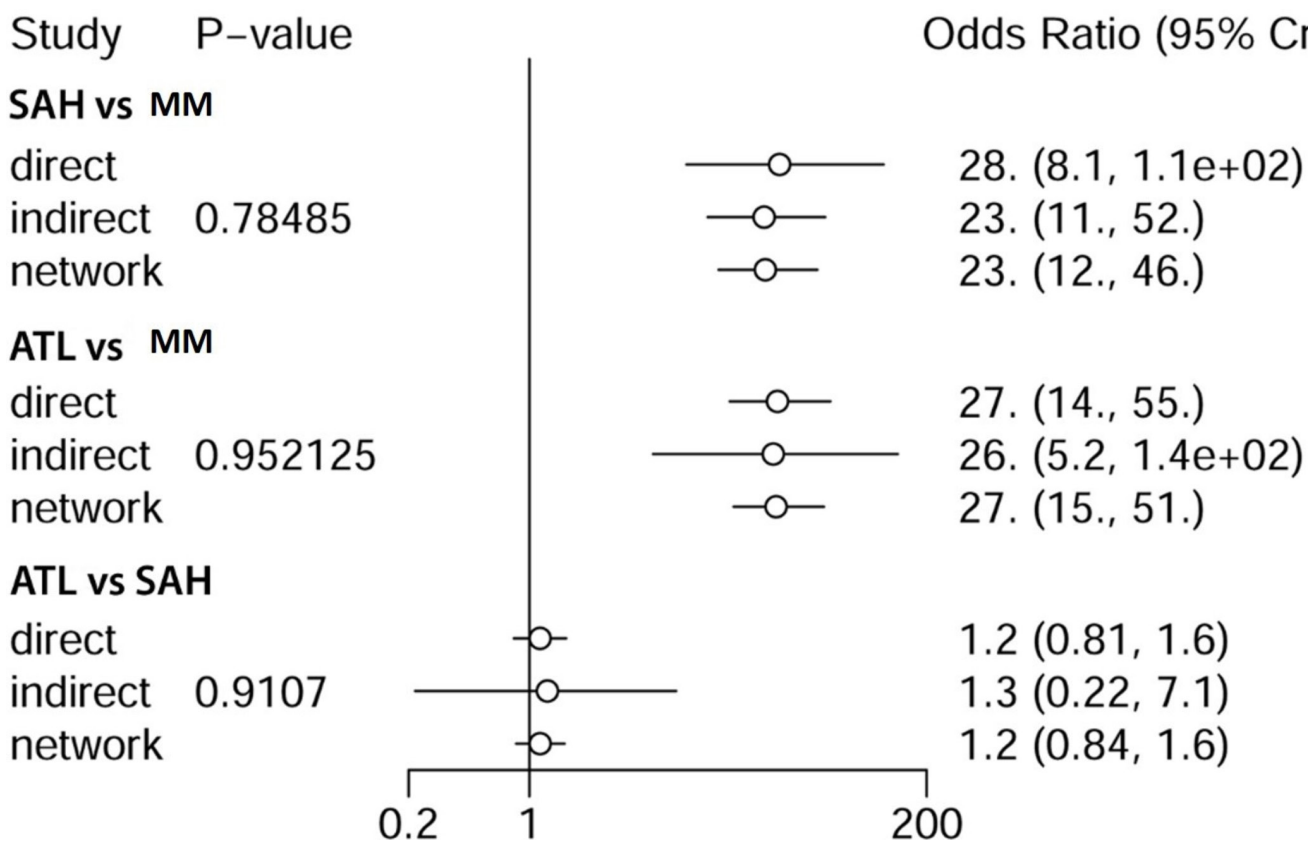

Figure 6 Forest plot for the direct and the indirect comparisons and network meta-analysis. There were no significant differences between direct and indirect comparisons for the three pairwise comparisons (ATL vs SAH, ATL vs MM, and SAH vs MM) (all p>0.05). The values of the odds ratio are truncated after the decimal points if more than 10. ATL, anterior temporal lobectomy; Crl, credible interval; MM, medical management; SAH, selective amygdalohippocampectomy.

outcome measures such as neuropsychological performance and health-related quality of life.

In summary, we have included direct and indirect evidence from the literature to evaluate seizure-free outcome of ATL versus SAH. We have used NMA to incorporate all relevant evidence from the literature so as to provide a more precise estimate of the treatment effect compared with using direct evidence of pairwise comparison of ATL versus SAH. We showed that direct evidence, indirect evidence and NMA did not identify a difference in seizure-free outcome of ATL versus SAH in TLE, suggesting that in patients with mesial TLE, ATL is not superior to SAH in achieving seizure-free outcome. However, there are methodological limitations in the existing literature, highlighting the need for a randomised controlled trial so as to provide a definitive answer on the effectiveness of ATL versus SAH.

Acknowledgements The authors would like to thank Alanna Marson (librarian at The Hospital for Sick Children) for assisting with the literature search.

Contributors EW and PJ planned the study. PJ and EW performed the systematic review and meta-analysis. PJ and EW wrote the first draft of the manuscript. PJ, GT, $\mathrm{CS}, \mathrm{BS}$ and EW provided inputs for the revision of the first draft of the manuscript and approved the final version.

Funding The authors have not declared a specific grant for this research from any funding agency in the public, commercial or not-for-profit sectors.

Competing interests None declared.

Patient consent Not required.

Provenance and peer review Not commissioned; externally peer reviewed.

(c) Article author(s) (or their employer(s) unless otherwise stated in the text of the article) 2018. All rights reserved. No commercial use is permitted unless otherwise expressly granted.

\section{REFERENCES}

1 Tatum WO. Mesial temporal lobe epilepsy. J Clin Neurophysiol 2012;29:356-65.

2 Engel J, McDermott MP, Wiebe S, et al. Early surgical therapy for drug-resistant temporal lobe epilepsy: a randomized trial. JAMA 2012;307:922-30.

3 Wiebe S, Blume WT, Girvin JP, et al. A randomized, controlled trial of surgery for temporal-lobe epilepsy. N Engl J Med 2001;345:311-8.
4 Hu WH, Zhang C, Zhang K, et al. Selective amygdalohippocampectomy versus anterior temporal lobectomy in the management of mesial temporal lobe epilepsy: a metaanalysis of comparative studies. J Neurosurg 2013;119:1089-97.

5 Josephson CB, Dykeman J, Fiest KM, et al. Systematic review and meta-analysis of standard vs selective temporal lobe epilepsy surgery. Neurology 2013;80:1669-76.

6 Kuang Y, Yang T, Gu J, et al. Comparison of therapeutic effects between selective amygdalohippocampectomy and anterior temporal lobectomy for the treatment of temporal lobe epilepsy: a meta-analysis. Br J Neurosurg 2014;28:374-7.

7 Jansen JP, Fleurence R, Devine $B$, et al. Interpreting indirect treatment comparisons and network meta-analysis for health-care decision making: report of the ISPOR Task Force on Indirect Treatment Comparisons Good Research Practices: part 1. Value Health 2011;14:417-28.

8 The Nordic Cochrane Centre, The Cochrane Collaboration. Review Manager (RevMan). 5.2. Copenhagen: The Nordic Cochrane Centre, The Cochrane Collaboration, 2012.

9 Lu G, Ades AE. Combination of direct and indirect evidence in mixed treatment comparisons. Stat Med 2004;23:3105-24.

10 Balshem $\mathrm{H}$, Helfand M, Schünemann HJ, et al. GRADE guidelines: 3. Rating the quality of evidence. J Clin Epidemiol 2011;64:401-6.

11 Shea BJ, Grimshaw JM, Wells GA, et al. Development of AMSTAR: a measurement tool to assess the methodological quality of systematic reviews. BMC Med Res Methodol 2007;7:10.

12 Dupont S, Tanguy ML, Clemenceau S, et al. Long-term prognosis and psychosocial outcomes after surgery for MTLE. Epilepsia 2006;47:2115-24.

13 Elsharkawy AE, Alabbasi AH, Pannek H, et al. Long-term outcome after temporal lobe epilepsy surgery in 434 consecutive adult patients. J Neurosurg 2009;110:1135-46.

14 Gül G, Yandim Kuşcu D, Özerden M, et al. Cognitive outcome after surgery in patients with mesial temporal lobe epilepsy. Noro Psikiyatr Ars 2017;54:43-8.

15 Lacruz ME, Alarcón G, Akanuma N, et al. Neuropsychological effects associated with temporal lobectomy and amygdalohippocampectomy depending on Wada test failure. J Neurol Neurosurg Psychiatry 2004;75:600-7.

16 Mansouri A, Fallah A, McAndrews MP, et al. Neurocognitive and seizure outcomes of selective amygdalohippocampectomy versus anterior temporal lobectomy for mesial temporal lobe epilepsy. Epilepsy Res Treat 2014;2014:1-8.

17 Ozkara C, Uzan M, Benbir G, et al. Surgical outcome of patients with mesial temporal lobe epilepsy related to hippocampal sclerosis. Epilepsia 2008;49:696-9.

18 Sindou M, Guenot M, Isnard J, et al. Temporo-mesial epilepsy surgery: outcome and complications in 100 consecutive adult patients. Acta Neurochir 2006;148:39-45.

19 Smyth MD, Limbrick DD, Ojemann JG, et al. Outcome following surgery for temporal lobe epilepsy with hippocampal involvement in preadolescent children: emphasis on mesial temporal sclerosis. J Neurosurg 2007;106:205-10.

20 Mathon B, Bielle F, Samson S, et al. Predictive factors of long-term outcomes of surgery for mesial temporal lobe epilepsy associated with hippocampal sclerosis. Epilepsia 2017; 58:1473-85. 
21 Harbord MG, Manson Jl. Temporal lobe epilepsy in childhood: reappraisal of etiology and outcome. Pediatr Neurol 1987;3:263-8.

22 Bien CG, Schulze-Bonhage A, Soeder BM, et al. Assessment of the long-term effects of epilepsy surgery with three different reference groups. Epilepsia 2006;47:1865-9.

23 Guldvog B, Løyning Y, Hauglie-Hanssen E, et al. Surgical versus medical treatment for epilepsy. I. Outcome related to survival, seizures, and neurologic deficit. Epilepsia 1991;32:375-88.

24 Helmstaedter C, Kurthen M, Lux S, et al. Chronic epilepsy and cognition: a longitudinal study in temporal lobe epilepsy. Ann Neurol 2003;54:425-32.

25 McGlone J, Valdivia I, Penner M, et al. Quality of life and memory after vagus nerve stimulator implantation for epilepsy. Can J Neurol Sci 2008;35:287-96.

26 Vickrey BG, Hays RD, Rausch R, et al. Outcomes in 248 patients who had diagnostic evaluations for epilepsy surgery. Lancet 1995;346:1445-9.

27 Chou CC, Shih YH, Yen DJ, et al. Long-term health-related quality of life in drugresistant temporal lobe epilepsy after anterior temporal lobectomy. Epileptic Disord 2015; 17:177-83.

28 Hemb M, Palmini A, Paglioli E, et al. An 18-year follow-up of seizure outcome after surgery for temporal lobe epilepsy and hippocampal sclerosis. J Neurol Neurosurg Psychiatry 2013:84:800-5.

29 Jones JE, Blocher JB, Jackson DC. Life outcomes of anterior temporal lobectomy: seria long-term follow-up evaluations. Neurosurgery 2013;73:1018-25.

30 Tanriverdi T, Olivier A. Cognitive changes after unilateral corticoamygdalohippocampectomy unilateral selective-amygdalohippocampectomy mesial temporal lobe epilepsy. Turk Neurosurg 2007:17:91-9.

31 McLachlan RS, Rose KJ, Derry PA, et al. Health-related quality of life and seizure control in temporal lobe epilepsy. Ann Neurol 1997:41:482-9.

32 Mikati MA, Comair YG, Rahi A. Normalization of quality of life three years after temporal lobectomy: a controlled study. Epilepsia 2006:47:928-33.

33 Boucher O, Dagenais E, Bouthillier A, et al. Different effects of anterior temporal lobectomy and selective amygdalohippocampectomy on verbal memory performance of patients with epilepsy. Epilepsy Behav 2015;52:230-5.

34 Tang Y, Yu X, Zhou B, et al. Short-term cognitive changes after surgery in patients with unilateral mesial temporal lobe epilepsy associated with hippocampal sclerosis. J Clin Neurosci 2014;21:1413-8.

35 Assaf BA, Karkar KM, Laxer KD, et al. Magnetoencephalography source localization and surgical outcome in temporal lobe epilepsy. Clin Neurophysiol 2004;115:2066-76.

36 Schijns OE, Bien CG, Majores M, et al. Presence of temporal gray-white matter abnormalities does not influence epilepsy surgery outcome in temporal lobe epilepsy with hippocampal sclerosis. Neurosurgery 2011;68:98-107.

37 Yasuda CL, Tedeschi H, Oliveira EL, et al. Comparison of short-term outcome between surgical and clinical treatment in temporal lobe epilepsy: a prospective study. Seizure 2006; 15:35-40.

38 Markand ON, Salanova V, Whelihan E, et al. Health-related quality of life outcome in medically refractory epilepsy treated with anterior temporal lobectomy. Epilepsia 2000;41:749-59.

39 Arruda F, Cendes F, Andermann F, et al. Mesial atrophy and outcome after amygdalohippocampectomy or temporal lobe removal. Ann Neurol 1996:40:446-50.

40 Bate $\mathrm{H}$, Eldridge $\mathrm{P}$, Varma T, et al. The seizure outcome after amygdalohippocampectomy and temporal lobectomy. Eur J Neurol 2007:14:90-4.

41 Bujarski KA, Hirashima F, Roberts DW, et al. Long-term seizure, cognitive, and psychiatric outcome following trans-middle temporal gyrus amygdalohippocampectomy and standard temporal lobectomy. J Neurosurg 2013;119:16-23.

42 Clusmann H, Schramm J, Kral T, et al. Prognostic factors and outcome after different types of resection for temporal lobe epilepsy. J Neurosurg 2002;97:1131-41.

43 Mackenzie RA, Matheson J, Ellis M, et al. Selective versus non-selective temporal lobe surgery for epilepsy. J Clin Neurosci 1997;4:152-4

44 Mittal S, Montes JL, Farmer JP, et al. Long-term outcome after surgical treatment of temporal lobe epilepsy in children. J Neurosurg 2005;103:401-12.

45 Morino M, Uda T, Naito K, et al. Comparison of neuropsychological outcomes after selective amygdalohippocampectomy versus anterior temporal lobectomy. Epilepsy Behav 2006;9:95-100.
46 Nascimento FA, Gatto LA, Silvado C, et al. Anterior temporal lobectomy versus selective amygdalohippocampectomy in patients with mesial temporal lobe epilepsy. Arq Neuropsiquiatr 2016;74:35-43.

47 Paglioli E, Palmini A, Portuguez $M$, et al. Seizure and memory outcome following temporal lobe surgery: selective compared with nonselective approaches for hippocampal sclerosis. J Neurosurg 2006;104:70-8.

48 Renowden SA, Matkovic Z, Adams CB, et al. Selective amygdalohippocampectomy for hippocampal sclerosis: postoperative MR appearance. AJNR Am J Neuroradiol 1995;16:1855-61.

49 Sagher 0 , Thawani JP, Etame AB, et al. Seizure outcomes and mesial resection volumes following selective amygdalohippocampectomy and temporal lobectomy. Neurosurg Focus 2012;32:E8.

50 Schmeiser B, Wagner K, Schulze-Bonhage A, et al. Surgical treatment of mesiotemporal lobe epilepsy: which approach is favorable? Neurosurgery 2017:81:992-1004.

51 Tahir MZ, Sobani ZA, Quadri SA, et al. Establishment of a comprehensive epilepsy center in pakistan: initial experiences, results, and reflections. Epilepsy Res Treat 2012;2012:1-6.

52 Tanriverdi T, Olivier A, Poulin N, et al. Long-term seizure outcome after mesial temporal lobe epilepsy surgery: corticalamygdalohippocampectomy versus selective amygdalohippocampectomy. J Neurosurg 2008;108:517-24.

53 Tanriverdi T, Dudley RW, Hasan A, et al. Memory outcome after temporal lobe epilepsy surgery: corticoamygdalohippocampectomy versus selective amygdalohippocampectomy. J Neurosurg 2010;113:1164-75.

54 Wendling AS, Hirsch E, Wisniewski I, et al. Selective amygdalohippocampectomy versus standard temporal lobectomy in patients with mesial temporal lobe epilepsy and unilateral hippocampal sclerosis. Epilepsy Res 2013;104:94-104.

55 Kellett MW, Smith DF, Baker GA, et al. Quality of life after epilepsy surgery. J Neurol Neurosurg Psychiatry 1997;63:52-8.

56 Altshuler L, Rausch R, Delrahim S, et al. Temporal lobe epilepsy, temporal lobectomy, and major depression. J Neuropsychiatry Clin Neurosci 1999;11:436-43.

57 Dias LA, Angelis G, Teixeira WA, et al. Long-term seizure, quality of life, depression, and verbal memory outcomes in a controlled mesial temporal lobe epilepsy surgical series using portuguese-validated instruments. World Neurosurg 2017;104:411-7.

58 Gilliam F, Kuzniecky R, Meador K, et al. Patient-oriented outcome assessment after temporal lobectomy for refractory epilepsy. Neurology 1999;53:687-94.

59 Jones JE, Berven NL, Ramirez L, et al. Long-term psychosocial outcomes of anterior temporal lobectomy. Epilepsia 2002;43:896-903.

60 Kumlien E, Doss RC, Gates JR. Treatment outcome in patients with mesial temporal sclerosis. Seizure 2002;11:413-7.

61 Caldwell DM, Ades AE, Higgins JP. Simultaneous comparison of multiple treatments: combining direct and indirect evidence. BMJ 2005;331:897-900.

62 Schramm J, Lehmann TN, Zentner J, et al. Randomized controlled trial of 2.5-cm versus 3.5-cm mesial temporal resection in temporal lobe epilepsy--Part 1: intent-to-treat analysis. Acta Neurochir 2011;153:209-19.

63 Hoyt AT, Smith KA. Selective amygdalohippocampectomy. Neurosurg Clin N Am 2016;27:1-17

64 Drane DL, Loring DW, Voets NL, et al. Better object recognition and naming outcome with MRI-guided stereotactic laser amygdalohippocampotomy for temporal lobe epilepsy. Epilepsia 2015:56:101-13.

65 Lutz MT, Clusmann H, Elger CE, et al. Neuropsychological outcome after selective amygdalohippocampectomy with transsylvian versus transcortical approach: a randomized prospective clinical trial of surgery for temporal lobe epilepsy. Epilepsia 2004:45:809-16

66 Schramm J. Temporal lobe epilepsy surgery and the quest for optimal extent of resection: a review. Epilepsia 2008;49:1296-307

67 Ojemann GA, Dodrill CB. Verbal memory deficits after left temporal lobectomy for epilepsy. Mechanism and intraoperative prediction. J Neurosurg 1985;62:101-7.

68 Hermann BP, Wyler AR, Somes G, et al. Pathological status of the mesial temporal lobe predicts memory outcome from left anterior temporal lobectomy. Neurosurgery 1992;31:652-6. Discussion 656-657.

69 Wolf RL, Ivnik RJ, Hirschorn KA, et al. Neurocognitive efficiency following left temporal lobectomy: standard versus limited resection. J Neurosurg 1993;79:76-83. 